\title{
Pancreatoduodenectomy With Blood Vessel Reconstruction for a Huge Solid Pseudopapillary Tumor of the Pancreas: Report of a Case
}

\author{
Qiang Wei ${ }^{\mathrm{a}}$, Xiao Xuc ${ }^{\mathrm{a}}$, Xin Duan ${ }^{\mathrm{a}}$, Qi Ling ${ }^{\mathrm{a}}$, Qinghong Ke ${ }^{\mathrm{a}}$, Shusen Zheng, b
}

\begin{abstract}
Giant solid pseudopapillary tumor (SPPT) with superior mesenteric artery (SMA) and hepatic artery (HA) invaded is a relative contraindication for surgical resection. As the improvement of surgical technique these years, pancreatoduodenectomy combined with vascular reconstruction can be performed under this circumstance. Here, we report a case of a SPPT of the pancreas with blood vessels invaded. We report the case of a 19-year-old woman who suffered from SPPT and underwent pancreaticoduodenectomy with SMA reconstruction. The patient is being followed-up and in good condition until now. A review of the relevant literature is also presented. This case demonstrates that a giant SPPT with blood vessels invaded is a rare condition and surgical resection with SMA reconstruction is generally curative.
\end{abstract}

Keywords: Pancreaticoduodenectomy; SPPT; Reconstruction

\section{Introduction}

SPPT is a borderline tumor of the pancreas $(0.13-2.7 \%$ of all pancreatic tumors and $1-3 \%$ of exocrine pancreas neoplasms) which shows an "enigmatic" behavior on the clinical and molecular pattern [1]. SPPT was first described by Frantz in 1959 [2] and was designated as a solid pseudopapillary tumor of the pancreas by WHO in 1996 [3]. These tumors affect predominantly young females between 20 - 30 years of age $[1,4]$ and are of unclear pathogenesis and low malignancy

Manuscript accepted for publication May 10, 2012

${ }^{a}$ Key Lab of Combined Multi-Organ Transplantation, Ministry of Public Health, Division of Hepatobiliary and Pancreatic Surgery, Department of Surgery, First Affiliated Hospital, Zhejiang University School of Medicine, 79 Qingchun Road, Hangzhou, Zhejiang, China

${ }^{\mathrm{b}}$ Corresponding author: Shusen Zheng, Key Lab of Combined Multi-Organ Transplantation, Ministry of Public Health, Division of Hepatobiliary and Pancreatic Surgery, Department of Surgery, First Affiliated Hospital, Zhejiang University School of Medicine, 79 Qingchun Road, Hangzhou, Zhejiang, China. Email: zyzss@zju.edu.cn

doi: http://dx.doi.org/10.4021/jmc691w which with an overall 5 years survival rate of $98 \%$ and 10 year of $93 \%$ respectively after surgery [5].

Surgical resection offers an excellent chance for longterm survival, such as pancreaticoduodenectomy, local tumor resection, segmental pancreas resection, resection of pancreatic body and tail and spleen resection.

Pancreatic resection for pancreatic borderline and malignancy tumor such as SPPT is selected only if all gross tumors can be removed to increase the cure rate. As the pancreas is so close to the blood vessels include SMV, SMA and $\mathrm{HA}$, which make tumor resection being a great challenge. Radical cure by traditional surgical procedures may be difficult $[6,7]$. In order to achieve radical resection, pancreatoduodenectomy combined with vascular reconstruction as a type of curative surgery should be performed by excellent surgical technology which leads to less postoperative complication $[8,9]$.

We present the case of a young girl who successfully underwent pancreaticoduodenectomy accompanied by SMA reconstruction in our center for an SPPT of the pancreatic head with blood vessels invasion.

\section{Case Report}

\section{Present history}

A 19-year-old girl was referred to our clinic for the management of a mass located in the right upper abdomen. The mass was discovered during the past 7 months and was confirmed by abdominal computer tomography (CT) scan of the local hospital for a minor traffic accident 9 days ago. For further treatment, she was admitted.

\section{Physical examination}

Vital signs are stable. A huge mass can be touched in right upper abdomen.

\section{Laboratory data}

Blood investigation and tumor markers (CEA, CA-153, CA- 


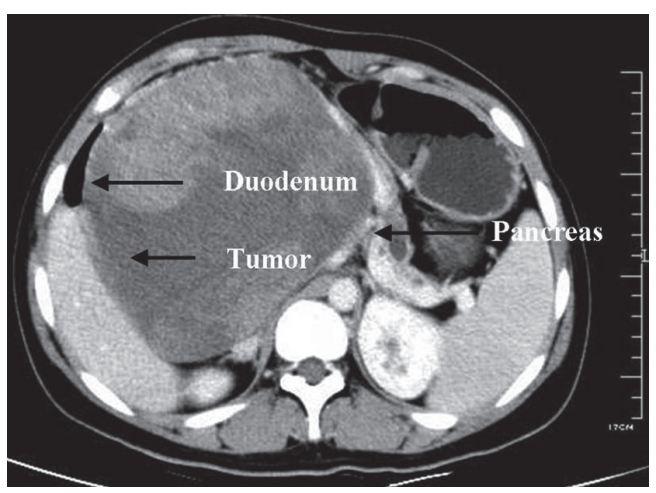

Figure 1. CT scan showing a cystic mass $(12.2 \mathrm{~cm}$ * 15.4 $\mathrm{cm})$ at the head of the pancreas in a 19-year-old girl with duodenum and pancreas compressed.

199, and CA-125) were within normal limits.

\section{Image findings}

In our hospital, we investigated the computed tomography angiography (CTA) scan, which revealed that the mass was located in the right upper abdomen and the celiac, gastroduodenal artery and superior mesenteric artery (SMA) were tumor feeding vessels (Fig.1, 2).

Magnetic resonance cholangiopancreatography (MRCP) showed a space-occupying lesion of maximum diameter of $18.2 \mathrm{~cm}$ located in the right upper abdomen, with compressed and displacement of the common bile duct and dilation of pancreatic body and tail duct (Fig. 3).

For diagnosis, a B ultrasound-guided puncture of the mass was performed before surgery and the pathology showed small round cell malignancies.

\section{Treatment}

She underwent pancreaticoduodenectomy (Whipple procedure) done by our surgical team. In the operation, we found duodenum and pancreas were compressed and the SMA and SMV were both invaded and came through the tumor. So the SMA and SMV reconstruction were performed. Especially, we reconstruct the SMA by self great saphenous vein transplantation. The resection material was sent to the pathology department (Fig. 4A, B).

On gross examination, a well-circumscribed, capsulated, round, gray-purple colored tumor was identified in the pancreatic head which contains varying amounts of necrosis, hemorrhage and cystic change (Fig. 4C). Microscopic analysis revealed the diagnosis of solid pseudo-papillary tumor. Results of immunoperoxidase stains showed tumor cells that were positive for CK, CD56, CD10 and $\beta$-catenin. In the evaluation of Ki-67, less than $2 \%$ of the tumor cells showed nuclear proliferative activity.

The patient was subsequently discharged after 2 weeks. CT scan performed 6 months postoperatively showed no evidence of disease recurrence.

\section{Discussion}

SPPT was first described by Frantz in 1959 [2]. Frantz tumor, solid and cystic tumor, solid and papillary epithelial neoplasm, papillary-cystic neoplasm of the pancreas, and Hamoudi tumor have been used to describe this rare lesion $[10,11]$. From 1996, the tumor was designated as a solid pseudopapillary tumor of the pancreas by WHO [3].

Patients with SPTT usually experience epigastric discomfort, nausea, weight loss, pain which are vague and unspecific, and they may have a palpable abdominal mass [10, $12,13]$. Some patients have no clinical symptoms but are discovered by common physical examination. In the present case, the patient remained asymptomatic although an increase of the lesion up to more than $18 \mathrm{~cm}$ diameter and SMV compression was assessed when she came to our department.

Most of the tumor was $5-10 \mathrm{~cm}$ in size while in our case the rare size of $19 \mathrm{~cm}$ was observed [5]. SPTT cells are typically positive for vimentin, CD10, CD56 and alpha1-antitrypsin and may reveal focal immunoreactivity for neuron-specific enolase, cytokeratin and synaptophysin, demonstrate abnormal nuclear localization of beta-catenin and may express galectin-3, all of which are useful in dif-

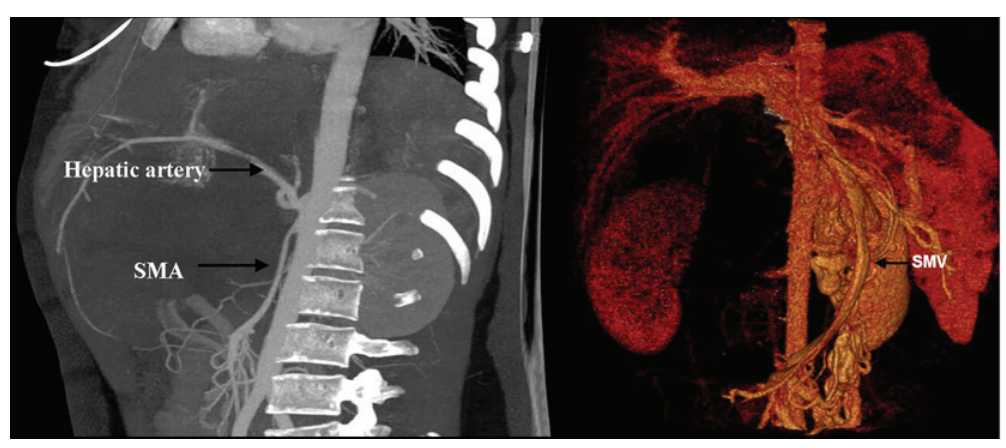

Figure 2. CTA showed that SMA was invaded. 


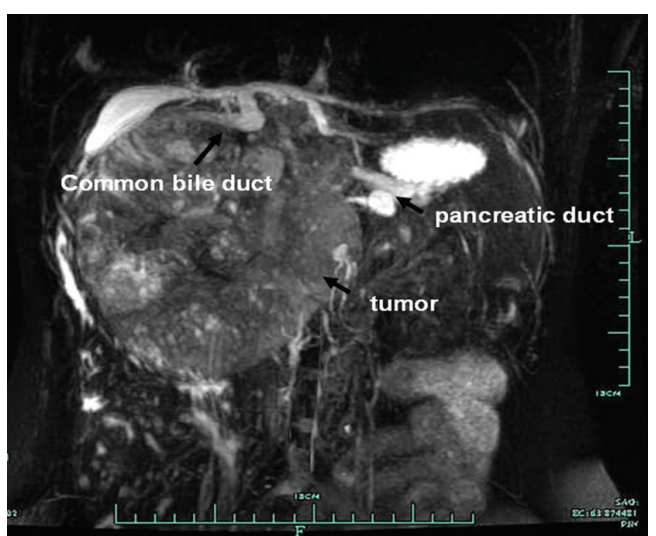

Figure 3. MRCP showed a space-occupying lesion (15.9 $\mathrm{cm} * 14.6 \mathrm{~cm}$ ) located in the right upper abdomen, with compressed and displacement of the common bile duct and dilation of pancreatic body and tail duct.

ferentiating them from endocrine pancreatic tumor cells $[10,14,15]$. In the present case, immunoperoxidase stains showed SPTT cells were positive for CK, Vimentin, CD56, $\mathrm{CD} 10$ and $\beta$-catenin, which confirmed that SPTT is not an endocrine origin.

Surgical resection is the first choice of treatment which including pancreaticoduodenectomy, local tumor resection, segmental pancreas resection, resection of pancreatic body and tail and spleen resection according to the location of the tumor $[16,17]$. Other adjuvant therapies, including chemotherapy and radiotherapy, had shown no demonstrable response [18]. Up to now, open laparotomy has been considered as the treatment of choice. To avoid metastatic spread, laparoscopic or laparotomic biopsy should not be performed in patients affected by SPTT $[19,20]$.

About $15 \%$ of cases of SPT have shown aggressive behavior consisting of extension into adjacent blood vessels and organs, local recurrence and distant metastasis [18, 21]. In our case, the tumor located in the pancreatic head with SMA invaded. So the reconstruction of blood vessels was performed. An excellent outcome has been achieved with 3 months follow-up at the time writing this article.

\section{Conclusion}

In this article we described a rare case of SMA involvement and blood vessels reconstruction was performed.

\section{Conflict of Interest Statement}

All the authors indicated that we have no financial relationship with the organization that sponsored the research. We have full control of all primary data and we all agree to allow the journal to review their data if requested. All of the authors have no conflict of interest.

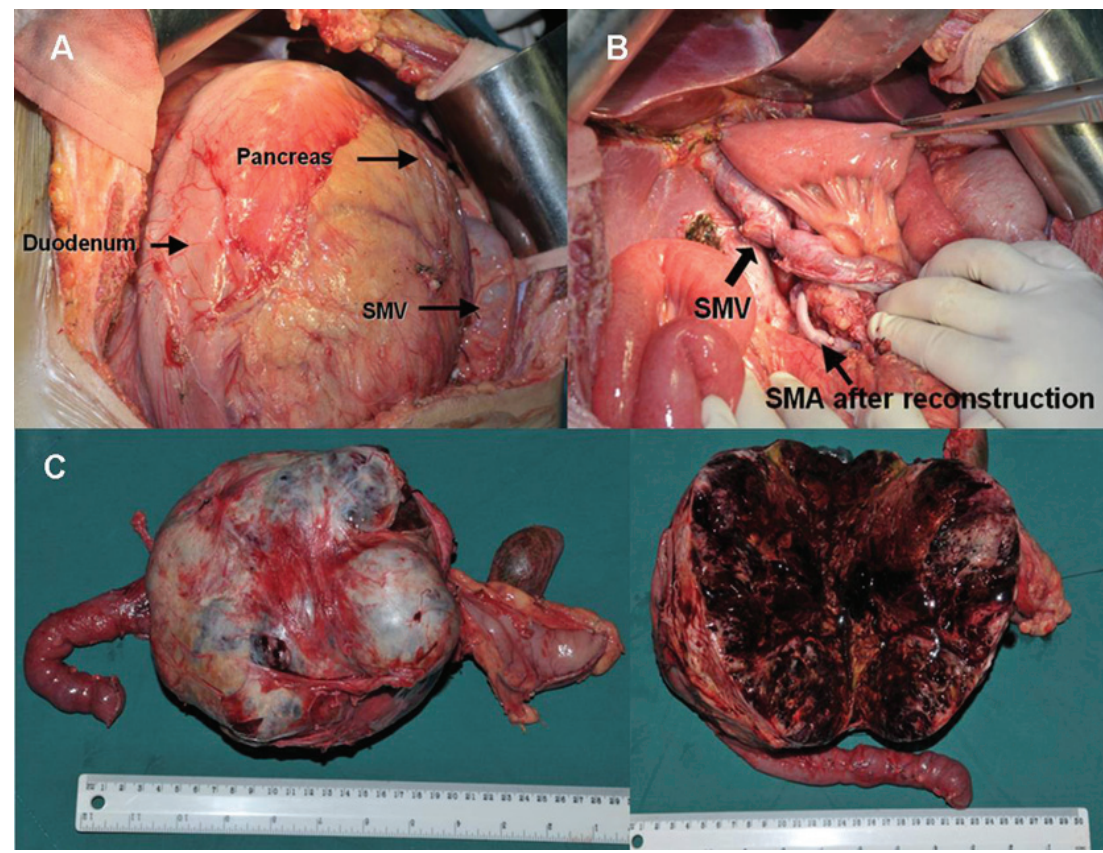

Figure 4. Gross appearance

A. The tumor located in the head of the pancreas with duodenum, pancreas and SMV compressed.

B. After reconstruction of blood vessels.

C \& D. Photograph of gross specimen showing a circumscribed, capsulated, round, gray-purple colored tumor was identified in the pancreatic head which contains varying amounts of necrosis, hemorrhage and cystic change. 


\section{References}

1. Crawford BE, 2nd. Solid and papillary epithelial neoplasm of the pancreas, diagnosis by cytology. South Med J. 1998;91(10):973-977.

2. Frantz VK. Tumor of the pancreas. In: Atlas of Tumor Pathology. Section 7, fascicles 27 and 28. Washington, DC, USA: Armed Forces Institute of Pathology; 1959:32-3.

3. Kloppel G, Solcia E, Longnecker DS, Capella C, Sobin LH. Histological typing of tumours of the exocrine pancreas. World Health Organization, International Histological Classification of Tumours. 2nd ed. Berlin: Springer-Verlag, 1996:11-20.

4. Yang F, Fu DL, Jin C, Long J, Yu XJ, Xu J, Ni QX. Clinical experiences of solid pseudopapillary tumors of the pancreas in China. J Gastroenterol Hepatol. 2008;23(12):1847-1851.

5. J AC, Lozano MD, Rotellar F, Marti P, Pedano N, Arredondo J, Bellver M, et al. Solid pseudopapillary tumor of the pancreas (SPPT). Still an unsolved enigma. Rev Esp Enferm Dig. 2010;102(12):722-728.

6. van Geenen RC, ten Kate FJ, de Wit LT, van Gulik TM, Obertop H, Gouma DJ. Segmental resection and wedge excision of the portal or superior mesenteric vein during pancreatoduodenectomy. Surgery. 2001;129(2):158163.

7. Nakao A, Kaneko T, Takeda S, Inoue S, Harada A, Nomoto S, Ekmel T, et al. The role of extended radical operation for pancreatic cancer. Hepatogastroenterology. 2001;48(40):949-952.

8. Ishikawa O, Ohigashi H, Yamada T, Sasaki Y, Imaoka S, Nakaizumi A, Uehara H, et al. Radical resection for pancreatic cancer. Acta Gastroenterol Belg. 2002;65(3):166170.

9. Cusack JC, Jr., Fuhrman GM, Lee JE, Evans DB. Managing unsuspected tumor invasion of the superior mesenteric-portal venous confluence during pancreaticoduodenectomy. Am J Surg. 1994;168(4):352-354.

10. Coleman KM, Doherty MC, Bigler SA. Solid-pseudopapillary tumor of the pancreas. Radiographics. 2003;23(6):1644-1648.

11. Cantisani V, Mortele KJ, Levy A, Glickman JN, Ricci P, Passariello R, Ros PR, et al. MR imaging features of solid pseudopapillary tumor of the pancreas in adult and pediatric patients. AJR Am J Roentgenol. 2003;181(2):395401.

12. Kawamoto S, Scudiere J, Hruban RH, Wolfgang CL, Cameron JL, Fishman EK. Solid-pseudopapillary neoplasm of the pancreas: spectrum of findings on multidetector CT. Clin Imaging. 2011;35(1):21-28.

13. Dong DJ, Zhang SZ. Solid-pseudopapillary tumor of the pancreas: CT and MRI features of 3 cases. Hepatobiliary Pancreat Dis Int. 2006;5(2):300-304.

14. Tang LH, Aydin H, Brennan MF, Klimstra DS. Clinically aggressive solid pseudopapillary tumors of the pancreas: a report of two cases with components of undifferentiated carcinoma and a comparative clinicopathologic analysis of 34 conventional cases. Am J Surg Pathol. 2005;29(4):512-519.

15. Geers C, Moulin P, Gigot JF, Weynand B, Deprez P, Rahier J, Sempoux C. Solid and pseudopapillary tumor of the pancreas--review and new insights into pathogenesis. Am J Surg Pathol. 2006;30(10):1243-1249.

16. Tipton SG, Smyrk TC, Sarr MG, Thompson GB. Malignant potential of solid pseudopapillary neoplasm of the pancreas. Br J Surg. 2006;93(6):733-737.

17. Gedaly R, Toledano A, Millan G, Essenfeld H, Zambrano VJ. Treatment of liver metastases from a solid pseudopapillary tumor of the pancreas. J Hepatobiliary Pancreat Surg. 2006;13(6):587-590.

18. Martin RC, Klimstra DS, Brennan MF, Conlon KC. Solid-pseudopapillary tumor of the pancreas: a surgical enigma? Ann Surg Oncol. 2002;9(1):35-40.

19. Fais PO, Carricaburu E, Sarnacki S, Berrebi D, Orbach $\mathrm{D}$, Baudoin V, de Lagausie P. Is laparoscopic management suitable for solid pseudo-papillary tumors of the pancreas? Pediatr Surg Int. 2009;25(7):617-621.

20. Cavallini A, Butturini G, Daskalaki D, Salvia R, Melotti G, Piccoli M, Bassi C, et al. Laparoscopic pancreatectomy for solid pseudo-papillary tumors of the pancreas is a suitable technique; our experience with long-term follow-up and review of the literature. Ann Surg Oncol. 2011;18(2):352-357.

21. Lam KY, Lo CY, Fan ST. Pancreatic solid-cystic-papillary tumor: clinicopathologic features in eight patients from Hong Kong and review of the literature. World $\mathbf{J}$ Surg. 1999;23(10):1045-1050. 